\title{
Cosmological MHD simulation of a cooling flow cluster
}

\author{
Y. Dubois and R. Teyssier
}

\author{
Service d'Astrophysique, CEA/DSM/IRFU/SAp, Centre d'Études de Saclay, L'Orme des Merisiers, \\ 91191 Gif-sur-Yvette Cedex, France \\ e-mail: ydubois@cea.fr
}

Received 4 February 2008 / Accepted 13 March 2008

\section{ABSTRACT}

\begin{abstract}
Context. Various observations of magnetic fields in the intra-cluster medium (ICM), most of the time restricted to cluster cores, point towards a field strength of a few $\mu \mathrm{G}$ (synchrotron radiation from radio relics and radio halos, inverse Compton radiation in X-rays and Faraday rotation measure of polarised background sources). Both the origin and the spatial structure of galaxy cluster magnetic fields are still under debate. In particular, the radial profile of the magnetic field, from the core of clusters to their outskirts, is important for cosmic ray propagation within the cosmic web.

Aims. In this letter, we highlight the importance of cooling processes in amplifying the magnetic field in the core of galaxy clusters up to one order of magnitude above the typical amplification obtained for a purely adiabatic evolution.

Methods. We performed a "zoom" cosmological simulation of a $3 \mathrm{keV}$ cluster, including dark matter and gas dynamics, atomic cooling, UV heating, and star formation using the newly developed MHD solver in the AMR code RAMSES.

Results. Magnetic field amplification proceeds mainly through gravitational contraction. Shearing motions due to turbulence provide additional amplification in the outskirts of the cluster, while magnetic reconnection during mergers causes magnetic field dissipation in the core.

Conclusions. Cooling processes have a strong impact on the magnetic field structure in the cluster. First, due to the sharp rise of the gas density in the centre, gravitational amplification is significantly higher, when compared to the non-radiative run. Second, cooling processes cause shearing motions to be much stronger in the core than in the adiabatic case, leading to additional field amplification and no significant magnetic reconnection. Cooling processes are therefore essential for determining the magnetic field profile in galaxy clusters.
\end{abstract}

Key words. galaxies: clusters: general - galaxies: cooling flows - galaxies: magnetic fields - methods: numerical

\section{Introduction}

Clusters of galaxies are known to be magnetised (see review by Govoni \& Feretti 2004). The existence of magnetic fields has been determined either by direct methods like diffuse synchrotron radio or inverse Compton hard X-ray emission or by indirect methods like Faraday rotation measures (RM). They all suggest that $\mu \mathrm{G}$ fields lie in central regions of galaxy clusters up to several $10 \mu \mathrm{G}$ magnetic fields in large cooling flows clusters. Magnetic field strength can differ from one method to the next Direct methods usually capture large-scale fields averaged over large volumes, while RM are derived from the analysis of background point sources and are thus sensitive to small-scale variations (cold filaments, shear flows, shocks, galaxy stripping, galaxy winds, etc.). To shed light on the magnetic topology found in cosmic structures, it is of great interest to perform direct, self-consistent numerical simulations of galaxy clusters. Magnetic fields in clusters are also important for determining the deflection angle of ultra-high energy cosmic rays, since they probably host the source of these cosmic rays (see Lemoine 2005; Kotera \& Lemoine 2008; Globus et al. 2007).

Simulations of galaxy clusters with magnetic fields have been performed using smooth particle hydrodynamics (SPH) codes (Dolag et al. 1999; Dolag 2000; Dolag et al. 2005), gridbased codes (Roettiger et al. 1999; Miniati et al. 2001; Sigl et al. 2004; Asai et al. 2007), and adaptive mesh refinement (AMR) codes (Brüggen et al. 2005), using both cosmological simulations (Dolag et al. 1999; Miniati et al. 2001; Sigl et al. 2004; Dolag et al. 2005) and idealised simulations (Roettiger et al. 1999; Asai et al. 2007). In this letter, we report the first cosmological simulation with AMR to include atomic cooling, UV heating, and star formation physics, with a full treatment of the ideal MHD equations. We have also performed a reference adiabatic run to compare our results with previous works and to point out the differences with the radiative case.

\section{Simulations}

We performed a "zoom" cosmological simulation of a galaxy cluster using the AMR code RAMSES (Teyssier 2002). Gas dynamics was computed using a second-order unsplit Godunov scheme for the ideal MHD equations (Teyssier et al. 2006; Fromang et al. 2006), while collisionless dark matter particles are evolved using a particle-mesh solver. Gas cooling and heating were taken into account as source terms in the energy equation. The cooling and heating functions were computed for a primordial H and He plasma, using the Haardt \& Madau (1996) background model. Radiative losses lead to the formation of high density, (low temperature) regions, where stars are allowed to form according to a Schmidt law: $\dot{\rho}_{*}=\epsilon \rho / t_{\text {ff }}$ if $\rho>\rho_{0}$. The density threshold for star formation was set to $\rho_{0}=10^{5} \Omega_{\mathrm{b}} \rho_{\mathrm{c}}(z)$. The star formation efficiency was set to $\epsilon=5 \%$. The simulation comoving box length was chosen equal to $80 h^{-1} \mathrm{Mpc}$ with a $\Lambda$ CDM cosmology with $\Omega_{\mathrm{m}}=0.3, \Omega_{\Lambda}=0.7, \Omega_{\mathrm{b}}=0.045$, 

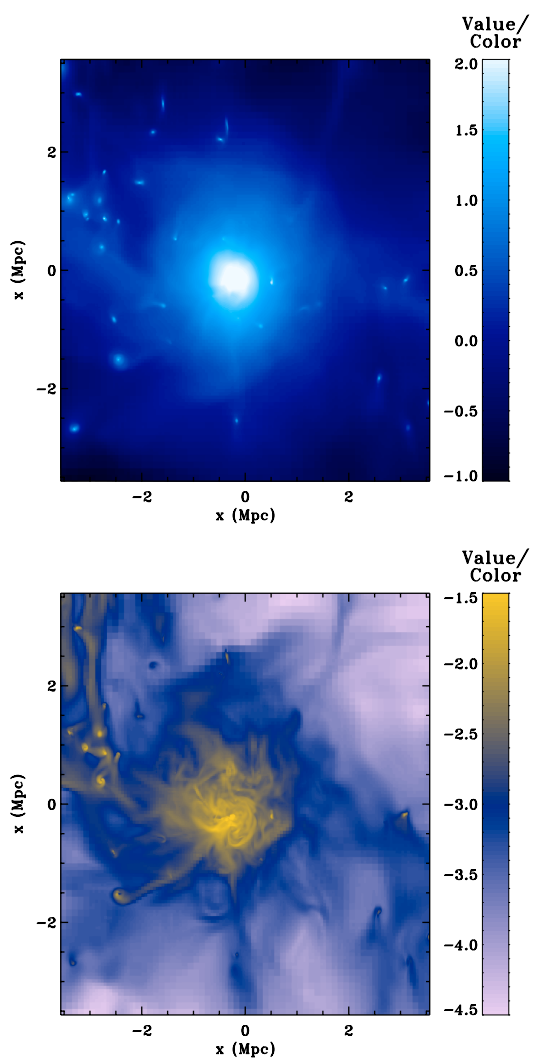

Fig. 1. Logarithm of the column density map of the gas in units of the mean baryons density (upper panel), and of the mass-averaged magnetic field amplitude in units of $\mu \mathrm{G}$ (bottom panel) at $z=0$.

$H_{0}=70 \mathrm{~km} \mathrm{~s}^{-1} \mathrm{Mpc}^{-1}$, and $\sigma_{8}=0.9$. A spherical region of radius $12.5 h^{-1} \mathrm{Mpc}$ around our simulated cluster was defined as our high-resolution region, with an effective resolution of $512^{3}$. A coarser grid with an effective resolution of $256^{3}$ was used to cover the inner $40 h^{-1} \mathrm{Mpc}$, and finally an even coarser $128^{3}$ grid was used to cover the whole box. The mass of dark matter particles on each coarse grid are $2.9 \times 10^{10} M_{\odot}, 3.6 \times 10^{9} M_{\odot}$, and $4.5 \times 10^{8} M_{\odot}$. Only the finest grid was allowed to trigger new refinements during the course of the simulation, up to 7 additional levels of AMR cells leading to a maximum resolution of $1.2 h^{-1} \mathrm{kpc}$. We used a quasi-Lagrangian criterion: each cell is individually refined if the number of dark matter particles exceeds 8 , or if the baryonic mass exceeds 8 times the initial high-resolution mass resolution. We solved the full set of ideal MHD equations using a new scheme based on a Godunov implementation of constrained transport and presented in Teyssier et al. (2006) and Fromang et al. (2006) and we used the HLLD Riemann solver from Miyoshi \& Kusano (2005). The comoving magnetic field was set initially to a constant value, $B_{\mathrm{z}} \simeq 10^{-11} \mathrm{G}$, as suggested in Dolag et al. (2005), to reproduce the $\mu \mathrm{G}$ fields in cluster cores. With these parameters in the course of the simulation, the plasma $\beta=P_{\text {gas }} / P_{\text {mag }}$ never decreased below 1000: the dynamical effect of the magnetic field can therefore be considered as negligible, even in the core of our simulated cluster.

\section{Results}

Figure 1 shows the column density distribution of the gas and the mean magnetic field for the cooling run at $z=0$. Magnetic field amplitudes are strongly correlated with the density distribution with mass-averaged values of $B \sim 10^{-1} \mu \mathrm{G}$ in the

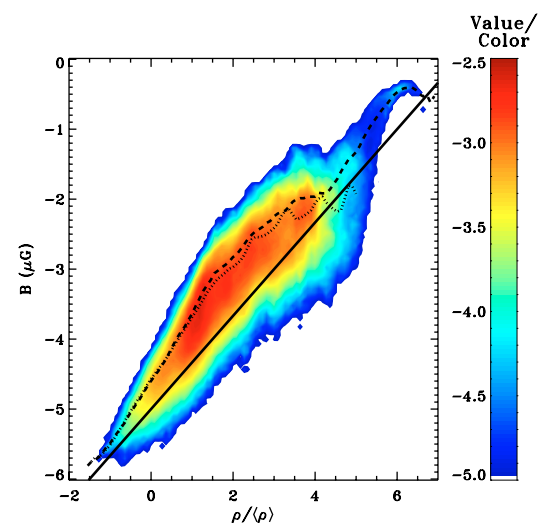

Fig. 2. Histogram of the mass fraction for the cooling run as a function of the normalised density and the magnetic amplitude at $z=0$. The black solid line is the $\rho^{2 / 3}$ collapse amplification, the averaged magnetic field as a function of the normalised density for the cooling case (dashed line) and the adiabatic case (dotted line) are also plotted.

cluster core, galaxies with $B \sim 10^{-2} \mu \mathrm{G}$ in satellite clumps, and $B \sim 10^{-3} \mu \mathrm{G}$ in filaments. We define the virial mass as $M_{200}=200 \times 4 \pi / 3 \rho_{\mathrm{c}} R_{200}^{3}$, where $\rho_{\mathrm{c}}$ is the critical density. For the adiabatic simulation, we found the following properties for our cluster at $z=0: R_{200}^{\text {ad }} \simeq 1 h^{-1} \mathrm{Mpc}, M_{200}^{\text {ad }} \simeq 2.7 \times 10^{14} h^{-1} M_{\odot}$, and $T_{\mathrm{X}}^{\mathrm{ad}} \simeq 3.4 \mathrm{keV}$. In the radiative case, we obtained $R_{200} \simeq$ $1.1 h^{-1} \mathrm{Mpc}, M_{200} \simeq 3.5 \times 10^{14} h^{-1} M_{\odot}$, and $T_{\mathrm{X}} \simeq 5.1 \mathrm{keV}$. The magnetic field amplification of a collapsing three-dimensional gas sphere with infinite conductivity is given by $B \propto \rho^{2 / 3}$, for the magnetic flux to be conserved. Thus a subsequent increase (or decrease) in the magnetic field with respect to this purely gravitational amplification should reveal other amplification or dissipation mechanisms. Figure 2 shows the mass-weighted histogram of the radiative simulation in the $\rho-|B|$ plane, within 2 virial radii around the cluster. For densities lower than $10^{4} \bar{\rho}$, the magnetic field amplification is one order of magnitude higher than for pure gravitational compression. As discussed in Dolag et al. (2005), this is likely due to shearing motions in the cluster atmosphere, caused by turbulence and frequent mergers. At higher densities, the radiative run diverges strongly from the adiabatic case. The gravitational compression due to the cooling flow provides additional field amplification in the high-density tail. As seen below, cooling also provides a sustained turbulent regime in the core and the corresponding additional field amplification. Based on the Zel'dovich approximation of gravitational dynamics, King \& Coles (2006) predict that a cosmological magnetic field should evolve as a $B \propto \rho^{0.87}$, due to anisotropic collapse in a Gaussian random field. This compares favourably with the low-density part of our simulation (see Fig. 2).

At higher density, the situation is more complex in the core of our simulated cluster. In the adiabatic case, the magnetic field amplitude decreases below the expected value for pure compression. The mean magnetic field is of the order of $10^{-2} \mu \mathrm{G}$, far below the typical values of magnetic field amplitude in observed cluster cores. It is also apparent in the magnetic field profile plotted in Fig. 3, for which a dip in the field strength is visible in the cluster core. While shear flows are able to sustain additional magnetic amplification in the outer parts of the cluster $r>150 h^{-1} \mathrm{kpc}$, we find the opposite behaviour in the cluster core where magnetic field is dissipated. According to Roettiger et al. (1999), this effect stems from magnetic reconnection occurring during merger events. Since we are not considering any microscopic process here and since the MHD turbulence scale 


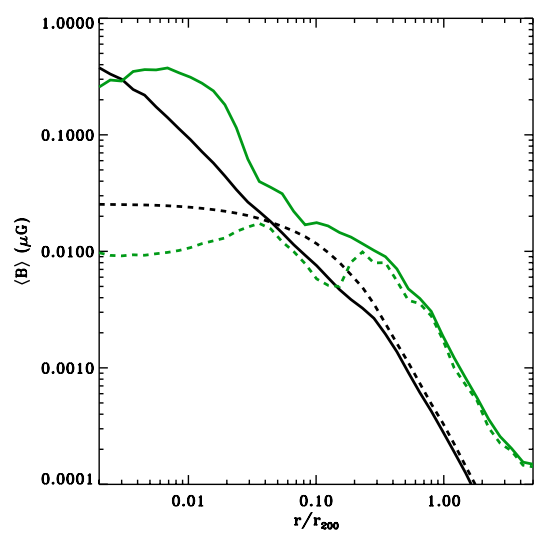

Fig. 3. Mean magnetic field as a function of the normalised radius. The black lines are the $\rho^{2 / 3}$ collapse amplification, the green lines denote the run values for the adiabatic (dotted) and cooling (solid) runs at $z=0$.

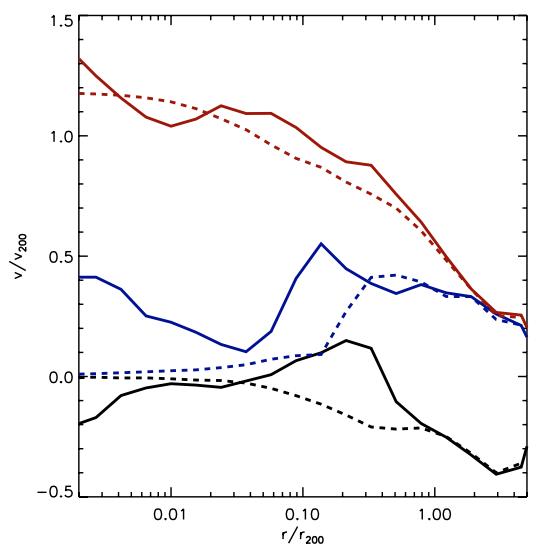

Fig. 4. Mean radial velocity (black), radial velocity dispersion (blue), and sound speed (red) for the adiabatic (solid) and the cooling (dashed) runs at $z=0$ in units of $V_{200}$ for the adiabatic case.

is far below our numerical resolution, this dissipation is caused by our numerical scheme that captures the weak solution of the ideal MHD equations. Although magnetic reconnection probably occurs in nature within converging flows, the exact amplitude of the dissipation is likely to depend on the microphysics. In the present numerical approach, results should depend strongly on the spatial resolution and on the numerical scheme used. It is however interesting to analyse the effect of cooling in this respect. As can be seen in Fig. 3, magnetic dissipation in the cluster core is suppressed and magnetic amplification now proceeds in the same way as in the outer parts, with gravitational compression and shearing motions. Only in the very centre (below $3 h^{-1} \mathrm{kpc}$, close to the resolution limit) do we see magnetic dissipation again.

To illustrate this point further, we show in Fig. 4 velocity profiles in the adiabatic and in the cooling cases. The radial velocity dispersion is a signature of turbulent motions, so it is no surprise to see that a strong velocity dispersion at a given radius corresponds to an excess of field amplification at the same radius (see Fig. 3). In the adiabatic case, turbulence is dissipated in the core, and magnetic dissipation occurs. In the cooling case, gravitational contraction resumes, as well as shearing motions, so that magnetic amplification is now more active in the core. The RM maps at $z=0$ of the adiabatic simulation and the cooling simulation shown in Fig. 5 differ strongly. In the adiabatic case, we obtain RM values of $30 \mathrm{rad} \mathrm{m}^{-2}$ in the cluster centre, whereas in the radiative case, we reach $1000 \mathrm{rad} \mathrm{m}^{-2}$ in the very centre
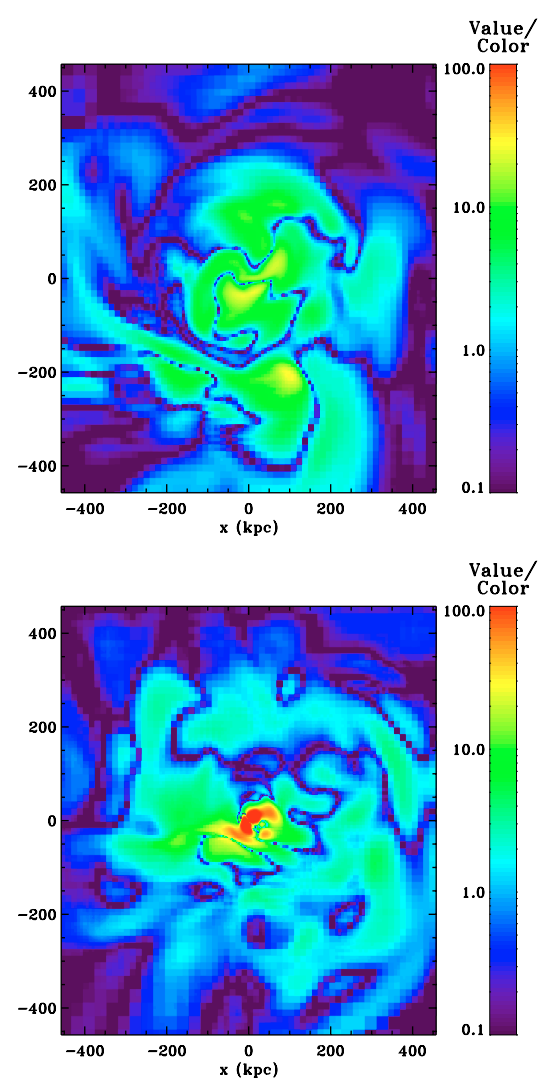

Fig. 5. $|\mathrm{RM}|$ maps colour scale of the cluster core for the adiabatic run (left panel) and the cooling run (right panel) at $z=0$.

$\left(r<10 h^{-1} \mathrm{kpc}\right)$ and obtain RM values of $100 \mathrm{rad} \mathrm{m}^{-2}$ in the core. The RM results of the cooling simulation are marginally consistent with the Clarke et al. (2001) sample $\left(200 \mathrm{rad} \mathrm{m}^{-2}\right)$ and also with the maximum values found in the cluster sample of Taylor et al. (2002) (up to $1800 \mathrm{rad} \mathrm{m}^{-2}$ for the hot gas cluster). The magnetic field in the outer part of our cluster is on the weak side of the Clarke et al. (2001) sample. It could result from differences in cluster mass or from our somewhat arbitrary choice of initial magnetic field. Note that we can increase the initial amplitude up to 10 times while remaining in the linear regime $(\beta \geq 10)$, so that all our results can be re-normalised by this factor.

\section{Conclusion and discussion}

There are noticeable differences in the magnetic field characteristics between a galaxy cluster with an adiabatic evolution and a galaxy cluster with radiative cooling: the average magnetic field in the cluster core is significantly higher when a cooling flow is present, due to additional gravitational compression but also an increased level of turbulence in the core-driving shearing motions. The main consequence is that Faraday RM simulated maps agree more with observations in the cooling case, if the initial comoving magnetic field value is taken to be $10^{-11} \mathrm{G}$. In low-density regions, however, the magnetic field evolution in the radiative run is very close to the adiabatic case since dynamical properties of the gas turbulence are nearly the same. Note that the turbulent cascade is far from being fully resolved in our simulation, especially outside the cluster core where our resolution barely reaches $10 h^{-1} \mathrm{kpc}$, a much larger scale than the dissipation scale of MHD turbulence (e.g. Brunetti \& Lazarian 2007; 
Jones 2007). For these reasons our results should only be considered as lower estimates for the magnetic field strength.

As was already discussed in Roettiger et al. (1999) in the context of adiabatic simulations of idealised mergers, magnetic reconnection might be responsible for field dissipation in the cluster core. Since we are dealing with ideal MHD, magnetic dissipation occurs at the numerical level, so that we should be affected to some extent by the effect of numerical resolution. Moreover, this underlines the importance of the choice of the numerical code used, especially when one considers the fundamental differences between grid-based and particle-based codes. Using an MHD version of GADGET, Dolag et al. (2005) find a much larger magnetic field strength in their adiabatic run for a subset of their simulated particles. They report mean field values one order of magnitude higher than median field values, apparently contradicting our present result. Magnetic dissipation therefore appears much less efficient in the SPH case. The Lagrangian nature of this technique is radically different from grid-based codes. Since both codes lead to different magnetic diffusion terms, a proper comparison between numerical techniques on this stake would be very helpful to the community. Using the grid-based code ZEUS, Roettiger et al. (1999) report very similar results to ours, with strong field dissipation occurring in the converging part of the flow. One interesting outcome of the present work is that radiative cooling drastically changes the effect of magnetic reconnection, since turbulence and gravitational compression easily counterbalance the associated dissipation.

Nevertheless, this large additional amount of magnetic energy in the core of cooling clusters is a crucial step in determining the structure of the cosmological magnetic field. It has a direct consequence on the propagation of high-energy cosmic rays in the universe. Since we have no direct observations of magnetic fields outside of cluster cores, only cosmological numerical simulations can address this problem. Their weakness is that the initial magnetic field value must be normalised a posteriori in order to fit the observed values. We have shown that, for the same seed field, the final magnetic field strength in a cooling cluster core is one order of magnitude more than in the adiabatic case. Cooling processes are therefore important if one wants to describe the proper evolution of magnetic fields in the Universe.

\section{References}

Asai, N., Fukuda, N., \& Matsumoto, R. 2007, ApJ, 663, 816 Brüggen, M., Ruszkowski, M., Simionescu, A., Hoeft, M., \& Dalla Vecchia, C. 2005, ApJ, 631, L21

Brunetti, G., \& Lazarian, A. 2007, MNRAS, 378, 245

Clarke, T. E., Kronberg, P. P., \& Böhringer, H. 2001, ApJ, 547, L111

Dolag, K. 2000, in Constructing the Universe with Clusters of Galaxies Dolag, K., Bartelmann, M., \& Lesch, H. 1999, A\&A, 348, 351

Dolag, K., Grasso, D., Springel, V., \& Tkachev, I. 2005, J. Cosmol. AstroParticle Phys., 1, 9

Fromang, S., Hennebelle, P., \& Teyssier, R. 2006, A\&A, 457, 371

Globus, N., Allard, D., \& Parizot, E. 2007, ArXiv e-prints, 709

Govoni, F., \& Feretti, L. 2004, Int. J. Mod. Phys. D, 13, 1549

Haardt, F., \& Madau, P. 1996, ApJ, 461, 20

Jones, T. W. 2007, ArXiv e-prints, 708

King, E. J., \& Coles, P. 2006, MNRAS, 365, 1288

Kotera, K., \& Lemoine, M. 2008, Phys. Rev. D, 77, 023005

Lemoine, M. 2005, Phys. Rev. D, 71, 083007

Miniati, F., Jones, T. W., Kang, H., \& Ryu, D. 2001, ApJ, 562, 233

Miyoshi, T., \& Kusano, K. 2005, J. Computational Phys., 208, 315

Roettiger, K., Stone, J. M., \& Burns, J. O. 1999, ApJ, 518, 594

Sigl, G., Miniati, F., \& Enßlin, T. A. 2004, Phys. Rev. D, 70, 043007

Taylor, G. B., Fabian, A. C., \& Allen, S. W. 2002, MNRAS, 334, 769

Teyssier, R. 2002, A\&A, 385, 337

Teyssier, R., Fromang, S., \& Dormy, E. 2006, J. Computational Phys., 218, 44 